\title{
Design of decentralized PI control systems based on Nyquist stability analysis
}

\author{
Dan Chen, Dale E. Seborg* \\ Department of Chemical Engineering University of California, Santa Barbara, CA 93106, USA
}

Received 27 December 2001; received in revised form 29 March 2002; accepted 23 April 2002

\begin{abstract}
This paper presents a simple, but effective, design method for decentralized PI control systems with guaranteed closed-loop stability. Nyquist stability conditions are used to derive the stability region for each PI controller in terms of the controller parameters. A detuning factor for each loop is specified based on a diagonal dominance index. Then appropriate controller settings are determined using this index and the stability region. Simulation results for a variety of $2 \times 2,3 \times 3$, and $4 \times 4$ systems demonstrate that the proposed design method guarantees closed-loop stability and provides good set-point and load responses. (C) 2002 Elsevier Science Ltd. All rights reserved.
\end{abstract}

Keywords: Decentralized PI control; Nyquist stability analysis; Diagonal dominance; Stability; Region

\section{Introduction}

Despite the development of advanced process control techniques, proportional-integral (PI) control is still the most commonly used control technique in the process industries. The main reasons for this popularity are that PI controllers are often effective and are easy to implement and maintain by plant personnel. Decentralized (or multi-loop) PI control systems are widely used for MIMO control problems in spite of the development of multivariable control strategies such as Model Predictive Control. The design and tuning of single loop PI controllers have been extensively researched [1-3]. But most of these techniques cannot be directly applied to design decentralized MIMO control systems. The latter problem is a much more complicated problem due to process and control loop interactions.

One way to compensate for loop interactions is to use a detuning factor for each control loop. If a MIMO process model is available, several detuning schemes for decentralized PI controllers are available based on frequency domain analysis. Perhaps the most well-known method is the biggest log modulus tuning (BLT) method proposed by Luyben [4-6]. In the BLT method, individual PI controllers are first designed using the ZieglerNichols $(\mathrm{ZN})$ tuning rules, then a detuning factor is

\footnotetext{
* Corresponding author. Fax: + 1-805-893-4731.

E-mail address: seborg@engineering.ucsb.edu (D.E. Seborg).
}

used for all of the loops. The detuning factor is adjusted so that the biggest log modulus, a measure of how far the system is from closed-loop instability, has a specified value. This method provides reasonable preliminary controller settings with guaranteed closed-loop stability.

Other design methods have been developed for decentralized PI control systems based on Nyquist stability analysis and frequency response information. Ho et al. [7] developed a design method for decentralized PID control systems by shaping the Gershgorin bands, the MIMO version of the Nyquist curve, so that the gain and phase margin specifications for the Gershgorin bands can be satisfied at the gain and phase crossover frequencies of the diagonal elements. This method cannot guarantee closed-loop stability because only two points of the Gershgorin bands are specified. Also, the process model is assumed to be a second-order plus dead-time model. Lee et al. [8] extended the iterative continuous cycling method for SISO problem to decentralized PI controller tuning. Their method refined the Nyquist array method to provide less conservative stability conditions, and ultimate gains for decentralized tuning are determined. But closed-loop stability is still not guaranteed due to the nature of the continuous cycling method.

If the process model is not available, the relay autotuning approach [1,9] is a useful and simple method to obtain system frequency information for PI controller design. Because MIMO systems have an infinite number of ultimate points [10], the relay auto-tuning approach 
for SISO systems cannot be directly applied. Loh et al. $[11,12]$ and Shen and $\mathrm{Yu}[13]$ have proposed sequential relay-feedback tests to locate the ultimate points of a MIMO system and to design PI controllers. Halevi et al. $[10,14]$ used simultaneous relays in all of the control loops to obtain the ultimate point information. In both the sequential and simultaneous relay auto-tuning approaches, after the frequency response information has been obtained, the ZN [15] or modified ZN tuning rules $[9,16,17]$ can be used to tune the PI/PID controllers.

In this paper a new design method for decentralized PI control systems is proposed based on the idea of independent design and Nyquist stability analysis. In the independent design procedure, loop interactions and the overall stability are considered first, and each controller is then designed independently of each other. Thus there are two main steps in the proposed method. First, a stability region is obtained for the proportional gain and integral time of each PI controller. Then appropriate PI controller settings are chosen which are inside the stability regions; thus closed-loop stability is guaranteed. For systems that are not column diagonally dominant, a pre-compensator is required to achieve diagonal dominance $[18,19]$. In the proposed method, no assumption is made concerning the form of the linear process model, i.e., the model is not limited to secondorder plus dead-time models or to any other specific form. Only the frequency response for each input-output pair is required to determine the stability region for each PI controller.

The proposed design method provides a simple way to apply Nyquist array analysis for the design and tuning of decentralized PI control systems with guaranteed closed-loop stability. A related paper [20] describes a simple alternative approach but it does not guarantee closed-loop stability. These design methods can be extended to accommodate model uncertainty using new robust stability conditions $[21,22]$.

\section{Stability analysis for decentralized control systems}

Consider an $n \times n$ system, $\mathbf{G}(s)=\left[g_{k l}(s)\right]_{n \times n}$, controlled by a decentralized controller, $\mathbf{C}(s)=\operatorname{diag}\left\{c_{1}(\mathrm{~s}), \ldots, c_{n}(s)\right\}$. The block diagram of the decentralized feedback con- trol system is shown in Fig. 1. It is assumed that $\mathbf{G}(s)$ has been arranged so that the pairings of the inputs and outputs in the decentralized feedback system correspond to the diagonal elements of $\mathbf{G}(s)$.

Let the diagonal closed-loop system be represented as

$$
\begin{aligned}
& \tilde{\mathbf{H}}(s)=\tilde{\mathbf{G}}(s) \mathbf{C}(s)(\mathbf{I}+\tilde{\mathbf{G}}(s) \mathbf{C}(s))^{-1} \\
& =\operatorname{diag}\left\{h_{l}(s)\right\}=\operatorname{diag}\left\{\frac{g_{l l}(s) c_{l}(s)}{1+g_{l l}(s) c_{l}(s)}\right\}
\end{aligned}
$$

where $\tilde{\mathbf{G}}(s)$ is the diagonal subsystem of $\mathbf{G}(s)$ :

$$
\tilde{\boldsymbol{G}}(s)=\operatorname{diag}\left\{g_{11}(s), g_{22}(s), \ldots, g_{n n}(s)\right\}
$$

In the independent design method, each controller $c_{l}(\mathrm{~s})$ is designed for the corresponding diagonal element $g_{l l}(s)$ so that each diagonal closed-loop system is stable. But due to the process interactions, the stability of this diagonal subsystem does not necessarily guarantee the stability of the overall closed-loop system:

$\mathbf{H}(s)=\mathbf{G}(s) \mathbf{C}(s)(\mathbf{I}+\mathbf{G}(s) \mathbf{C}(s))^{-1}$

Some constraints on the individual controller designs should be satisfied in order to guarantee stability of the overall system. The $\mu$-interaction measure [23], which bounds the amplitude of $\tilde{h}_{l}(j \omega)$, gives a sufficient condition for stability. This measure is somewhat conservative because the phase information for $\tilde{\mathbf{H}}(j \omega)$ is not utilized. Lee et al. [8] proposed a phase stability condition which provides a less conservative stability condition when it is used together with the $\mu$-interaction measure. The $\mu$-interaction measure is very useful but the computation is rather complex because the structured singular value is involved. The phase stability bound makes it even more complicated.

The Nyquist array method [24,25] is considered in this paper because it provides much simpler conditions for stability, provided that the open-loop system is diagonally dominant. Consider the Nyquist curve $g_{l l}(j \omega) c_{l}(j \omega)$ and superimpose a circle of radius,

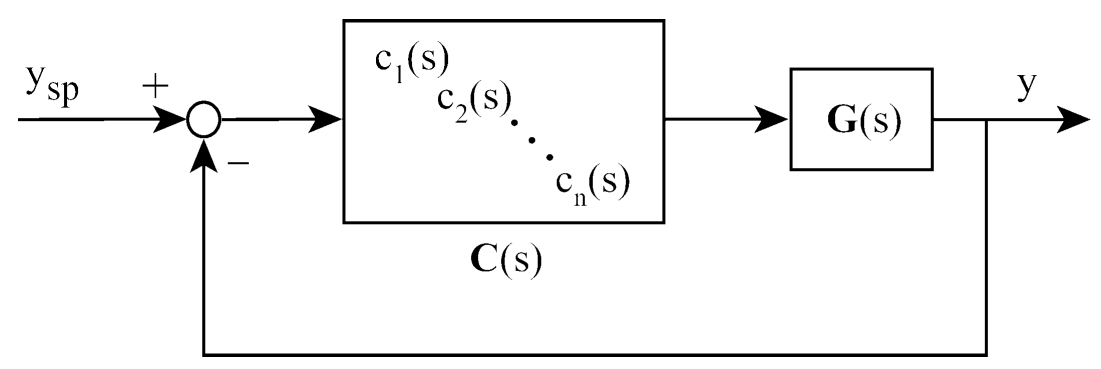

Fig. 1. General decentralized control system. 
$\sum_{k=1, k \neq l,}^{n}\left|g_{k l}(j \omega) c_{l}(j \omega)\right|$

This circle is referred to as Gershgorin circle. The band composed of Gershgorin circles for all frequencies is called the Gershgorin band. Gershgorin bands can be used to determine whether the closed-loop system remains stable in spite of the process interactions. The constraints on the Gershgorin bands for closed-loop stability can be obtained from the following theorem.

Theorem 1 [23]. Assume that $\boldsymbol{G}(\mathrm{s})$ and $\tilde{\boldsymbol{G}}(\mathrm{s})$ have the same number of right half-plane poles and that $\tilde{\boldsymbol{H}}(\mathrm{s})$ is stable. Then the closed-loop system $\boldsymbol{H}(\mathrm{s})$ is stable if

$\left|\tilde{h}_{l}(j \omega)\right|<\left[\sum_{k=1, k \neq 1}^{n}\left|g_{k l}(j \omega) c_{l}(j \omega)\right|\right]^{-1} \forall l, \omega$

Eq. (5) can be rewritten as

$\left|1+g_{l l}(j \omega) c_{l}(j \omega)\right|>\sum_{k=1, k \neq l}^{n}\left|g_{k l}(j \omega) c_{l}(j \omega)\right| \quad \forall l, \omega$

Eq. (6) presents column diagonal dominance criteria for the closed-loop system, $\mathbf{I}+\mathbf{G}(s) \mathbf{C}(s)$, while the left hand side of Eq. (6) defines the radii of the circles forming the Gershgorin bands [24]. Therefore, under the assumptions of Theorem 1, the overall closed-loop system $\mathbf{H}(s)$ is stable if column diagonal dominance is achieved for all loops at all frequencies.

The column diagonal dominance measure given by Eqs. (5) or (6) provides individual constraints for each single loop transfer function $\tilde{h}_{l}(j \omega)$, i.e., for each of the controllers $c_{l}(j \omega)$. It would be incorrect to view the $\mu$-interaction measure as less conservative than the column diagonal measure [23]. The $\mu$-interaction measure is a general method, which can consider a controller $\mathbf{C}(s)$ with block diagonal structure, while the column diagonal dominance measure is only valid for a diagonal controller $\mathbf{C}(s)$. However, for decentralized control systems the column diagonal measure is much simpler and easier to implement for system analysis and design, especially for fixed structure controllers such as PI or PID controllers.

Because most practical processes are open-loop stable, in this paper it is assumed that the system is open-loop stable. It is also assumed that a decentralized control system is used and the closed-loop system has column diagonal dominance. Then the Gershgorin band of each loop must be one of the two cases shown in Fig. 2.

If the open-loop Gershgorin band of the l-th loop is like Case II in Fig. 2, then this individual loop is not stable due to the encirclement of the critical point by the Nyquist locus. Thus as stated in Theorem 1, in order to achieve the closed-loop stability, the stability of the diagonal closed-loop system $\mathbf{H}(s)$ is required in addition to closed-loop column diagonal dominance.

\section{Stability region for decentralized PI systems}

In this section the stability of decentralized PI systems is analyzed by applying the stability condition given by Theorem 1.

Each element of the system frequency response can be expressed in terms of real and imaginary parts,

$g_{k l}(j \omega)=a_{k l}(\omega)+j b_{k l}(\omega)$

or in terms of the magnitude $r_{k l}$ and phase angle $\phi_{k l}$ :

$g_{k l}(j \omega)=r_{k l}(\omega) e^{j \phi_{k l}(\omega)}$
Case I

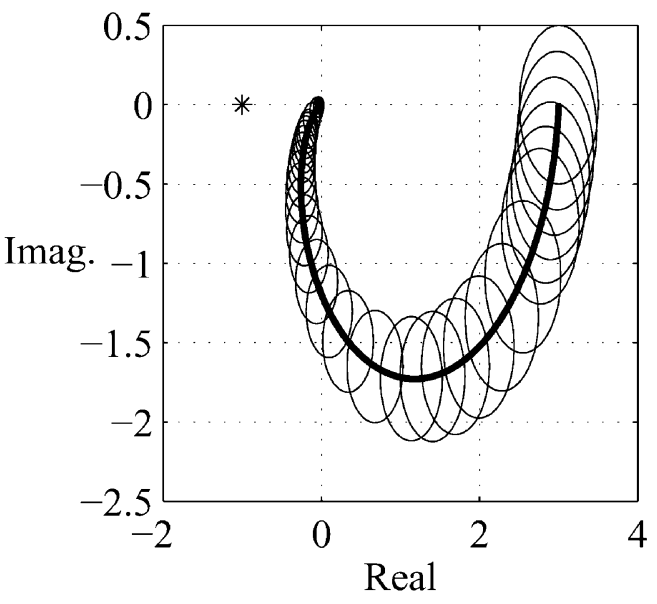

Case II

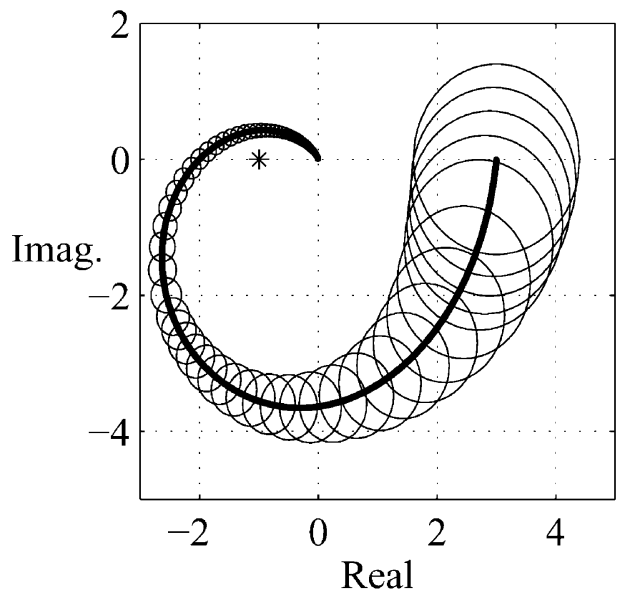

Fig. 2. Column diagonal dominance cases. 
Suppose that a decentralized PI control system $\mathbf{C}(s)=\operatorname{diag}\left\{c_{1}(s), \ldots, c_{\mathrm{n}}(s)\right\}$ is used to control the multivariable system, $\mathbf{G}(s)$, and that each $c_{l}(s)$ is a PI controller:

$c_{l}(s)=K_{c l}\left(1+\frac{1}{s \tau_{I l}}\right)$

or

$c_{l}(s)=K_{c l}+\frac{K_{l l}}{s}$

where $K_{I l} \triangleq K_{c l} / \tau_{I l}$ is called the integral gain.

\subsection{Column diagonal dominance}

Define

$R_{l}(\omega) \triangleq \sum_{k=1, k \neq l}^{n}\left|g_{k l}(j \omega)\right|$

$r_{l l}(\omega) \triangleq\left|g_{l l}(j \omega)\right|$

then the column diagonal dominance condition in Eq. (6) can be expressed as
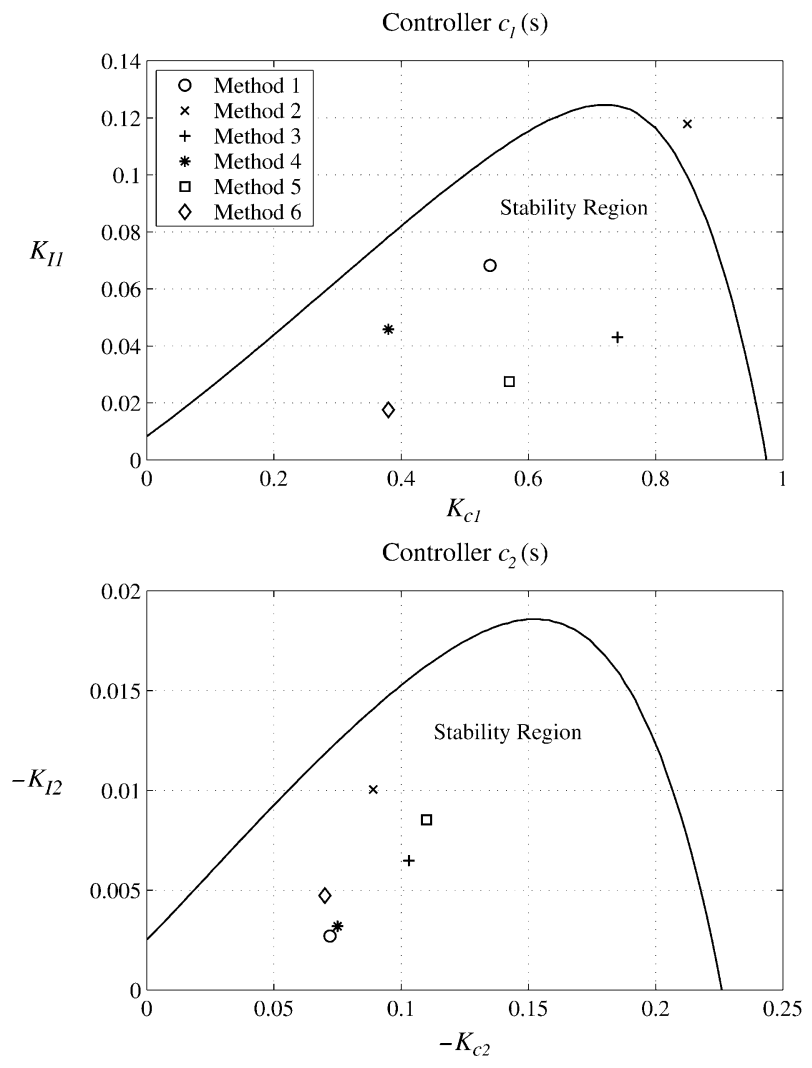

Fig. 3. Stability regions for PI controller settings: the Wood-Berry column example.

$$
\left|1+g_{l l}(j \omega) c_{l}(j \omega)\right|^{2}>\left|c_{l}(j \omega)\right|^{2} R_{l}^{2}(\omega) \quad \forall l, \omega
$$

Substituting (7) and (10) and using the definitions of (11) and (12), the inequality in (13) can be written as

$$
\begin{aligned}
& K_{c l}^{2}\left(r_{l l}^{2}(\omega)-R_{l}^{2}(\omega)\right)+K_{I l}^{2} \frac{r_{I l}^{2}(\omega)-R_{l}^{2}(\omega)}{\omega^{2}} \\
& +2\left(K_{c l} a_{l l}(\omega)+K_{I l} \frac{b_{l l}(\omega)}{\omega}\right)+1>0
\end{aligned}
$$

Therefore, a region can be obtained for $\left(K_{c l}, K_{I l}\right)$ of the $l$-th loop PI controller by applying condition (14) for $0 \leqslant \omega<\infty$. This region is referred to as the column diagonal dominance region for the $l$-th loop. For a MIMO system and a decentralized PI control system, if each PI controller has its parameters $\left(K_{c l}, K_{I l}\right)$ located inside the column diagonal dominance region, then the column diagonal dominance of the closed-loop system is achieved.

\subsection{Stability conditions for individual controllers}

As discussed in Section 1, each controller $c_{l}(s)$ should stabilize the corresponding diagonal element $g_{l l}(s)$ in order to achieve closed-loop stability for the overall system. Based on a Nyquist stability analysis for openloop stable SISO systems, Aström et al. [26] have derived the following parametric description of the stability boundary for a PI controller in terms of $K_{c l}$ and $K_{I l}$,

$$
\begin{aligned}
& K_{c l}=-\frac{a_{l l}(\omega)}{r_{l l}^{2}(\omega)} \\
& K_{I l}=-\omega \frac{b_{l l}(\omega)}{r_{l l}^{2}(\omega)}
\end{aligned}
$$

where $a_{l l}(\omega), b_{l l}(\omega)$, and $r_{l l}(\omega)$ are defined in Eqs. (7) and (8). By applying Eq. (15) for $0 \leqslant \omega<\infty$, an indivi-

\begin{tabular}{|c|c|c|c|c|c|}
\hline \multirow{2}{*}{$\begin{array}{l}\text { Tuning } \\
\text { method }\end{array}$} & \multicolumn{2}{|c|}{ Overhead } & \multicolumn{2}{|c|}{ Bottoms } & \multirow[t]{2}{*}{ Reference } \\
\hline & $K_{c 1}$ & $\tau_{I 1}$ & $K_{c 2}$ & $\tau_{I 2}$ & \\
\hline 1 & 0.54 & 7.92 & -0.072 & 26.70 & [13] \\
\hline 2 & 0.85 & 7.21 & -0.089 & 8.86 & [8] \\
\hline 3 & 0.74 & 17.20 & -0.103 & 15.90 & {$[8]^{\mathrm{a}}$} \\
\hline 4 & 0.38 & 8.29 & -0.075 & 23.60 & [4] \\
\hline 5 & 0.57 & 20.70 & -0.110 & 12.90 & [7] \\
\hline 6 & 0.38 & 21.60 & -0.070 & 14.80 & [7] \\
\hline
\end{tabular}
dual stability region boundary can be obtained for $\left(K_{c l}\right.$, $\left.K_{I l}\right)$ of the $l$-th PI controller.

Table 1

Controller settings for the Wood-Berry column example

a Tuning method originally developed in [30] 


\subsection{Overall stability condition}

In order to achieve the stability for overall system, each controller $c_{l}(s)$ should satisfy both the column diagonal dominance condition and the individual stability condition for $g_{l l}(s)$, i.e., the parameter settings $\left(K_{c l}\right.$, $\left.K_{I l}\right)$ of each controller should satisfy both Eqs. (14) and (15) for all frequencies. The column diagonal dominance region can be obtained from Eq. (14) by using a one-dimensional search. First a range for $K_{c l}$ is specified; then for each value of $K_{c l}$, a one-dimensional search is implemented for the $K_{I l}$ values which satisfy condition (14) for all frequencies. By applying Eq. (15), the individual stability boundary can be obtained for each $\left(K_{c l}, K_{I l}\right)$. Therefore, the overall stability region for $\left(K_{c l}, K_{I l}\right)$ of the $l$-th PI controller is the intersection of the column diagonal dominance region from Eq. (14) and the individual stability region from Eq. (15) for the $l$-th loop and for all frequencies.

\subsection{Wood-Berry distillation column model}

A simulation example is considered to demonstrate how to obtain the boundaries of stability regions for the parameter settings of a decentralized PI control system.

Wood and Berry [27] developed the following transfer function model of a pilot-scale distillation column that is used to separate a methanol-water mixture,

Table 2

14 cases of $3 \times 3$ systems

\begin{tabular}{|c|c|c|c|c|c|c|c|c|c|}
\hline \multirow[t]{2}{*}{ Case } & \multirow[t]{2}{*}{$g_{i i}(s)$} & \multirow{2}{*}{$\begin{array}{l}g_{i j}(s) \\
(i \neq j)\end{array}$} & \multirow[t]{2}{*}{$\lambda_{11}$} & \multirow[t]{2}{*}{$\phi_{u l}$} & \multirow[t]{2}{*}{$\mathrm{F}_{l}$} & \multicolumn{2}{|c|}{ Proposed design } & \multicolumn{2}{|c|}{ BLT design } \\
\hline & & & & & & $K_{c l}$ & $\tau_{I l}$ & $K_{c l}$ & $\tau_{I l}$ \\
\hline 1 & $\frac{2 \mathrm{e}^{-s}}{s+1}$ & $\frac{\mathrm{e}^{-2 s}}{1.2 s+1}$ & 1.5 & 0.135 & 0.466 & 0.269 & 2.44 & 0.296 & 4.44 \\
\hline 2 & $\frac{2 \mathrm{e}^{-s}}{s+1}$ & $\frac{\mathrm{e}^{-2 s}}{1.5 s+1}$ & 1.5 & 0.286 & 0.429 & 0.272 & 2.41 & 0.307 & 4.28 \\
\hline 3 & $\frac{2 \mathrm{e}^{-s}}{s+1}$ & $\frac{\mathrm{e}^{-2 s}}{2 s+1}$ & 1.5 & 0.452 & 0.387 & 0.274 & 1.87 & 0.313 & 4.21 \\
\hline 4 & $\frac{3 \mathrm{e}^{-s}}{s+1}$ & $\frac{\mathrm{e}^{-2 s}}{2 s+1}$ & 1.2 & 0.637 & 0.341 & 0.185 & 1.67 & 0.205 & 4.28 \\
\hline 5 & $\frac{5 \mathrm{e}^{-s}}{s+1}$ & $\frac{\mathrm{e}^{-2 s}}{2 s+1}$ & 1.07 & 0.783 & 0.304 & 0.112 & 1.57 & 0.122 & 4.32 \\
\hline 6 & $\frac{12 \mathrm{e}^{-s}}{s+1}$ & $\frac{\mathrm{e}^{-2 s}}{2 s+1}$ & 1.01 & 0.910 & 0.273 & 0.0469 & 1.50 & 0.0505 & 4.38 \\
\hline 7 & $\frac{2 \mathrm{e}^{-s}}{s+1}$ & $\frac{\mathrm{e}^{-2 s}}{0.9 s+1}$ & 1.5 & -0.0801 & 0.5 & 0.256 & 2.37 & 0.280 & 4.71 \\
\hline 8 & $\frac{2 \mathrm{e}^{-s}}{s+1}$ & $\frac{\mathrm{e}^{-2 s}}{0.8 s+1}$ & 1.5 & -0.170 & 0.5 & 0.244 & 2.36 & 0.273 & 4.82 \\
\hline 9 & $\frac{2 \mathrm{e}^{-s}}{s+1}$ & $\frac{\mathrm{e}^{-2 s}}{0.7 s+1}$ & 1.5 & -0.271 & 0.5 & 0.232 & 2.31 & 0.266 & 4.95 \\
\hline 10 & $\frac{2 \mathrm{e}^{-s}}{s+1}$ & $\frac{\mathrm{e}^{-2 s}}{0.6 s+1}$ & 1.5 & -0.385 & 0.5 & 0.220 & 2.28 & 0.259 & 5.08 \\
\hline 11 & $\frac{2 \mathrm{e}^{-s}}{s+1}$ & $\frac{\mathrm{e}^{-2 s}}{0.5 s+1}$ & 1.5 & -0.509 & 0.502 & 0.209 & 2.22 & 0.251 & 5.23 \\
\hline 12 & $\frac{2 \mathrm{e}^{-s}}{s+1}$ & $\frac{\mathrm{e}^{-2 s}}{0.4 s+1}$ & 1.5 & -0.647 & 0.537 & 0.211 & 2.09 & 0.244 & 5.39 \\
\hline 13 & $\frac{2 \mathrm{e}^{-s}}{s+1}$ & $\frac{\mathrm{e}^{-2 s}}{0.3 s+1}$ & 1.5 & -0.790 & 0.573 & 0.214 & 2.00 & 0.237 & 5.55 \\
\hline 14 & $\frac{2 \mathrm{e}^{-s}}{s+1}$ & $\frac{\mathrm{e}^{-2 s}}{0.1 s+1}$ & 1.5 & -1.051 & 0.638 & 0.221 & 1.84 & 0.227 & 5.81 \\
\hline
\end{tabular}


$\left[\begin{array}{c}X_{\mathrm{D}}(s) \\ X_{\mathrm{B}}(s)\end{array}\right]=\left[\begin{array}{cc}\frac{12.8 e^{-s}}{16.7 s+1} & \frac{-18.9 e^{-3 s}}{21 s+1} \\ \frac{6.6 e^{-7 s}}{10.9 s+1} & \frac{-19.4 e^{-3 s}}{14.4 s+1}\end{array}\right]\left[\begin{array}{l}R(s) \\ S(s)\end{array}\right]+\left[\begin{array}{c}\frac{3.8 \mathrm{e}^{-8.1 s}}{14.9 s+1} \\ \frac{4.9 e^{-3.4 s}}{13.2 s+1}\end{array}\right] F(s)$

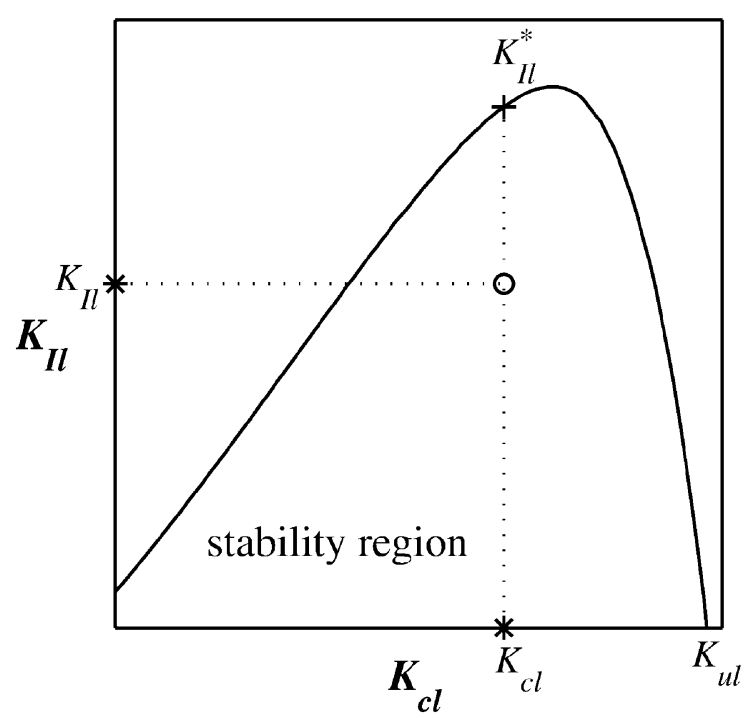

Fig. 4. Decentralized PI control system tuning.
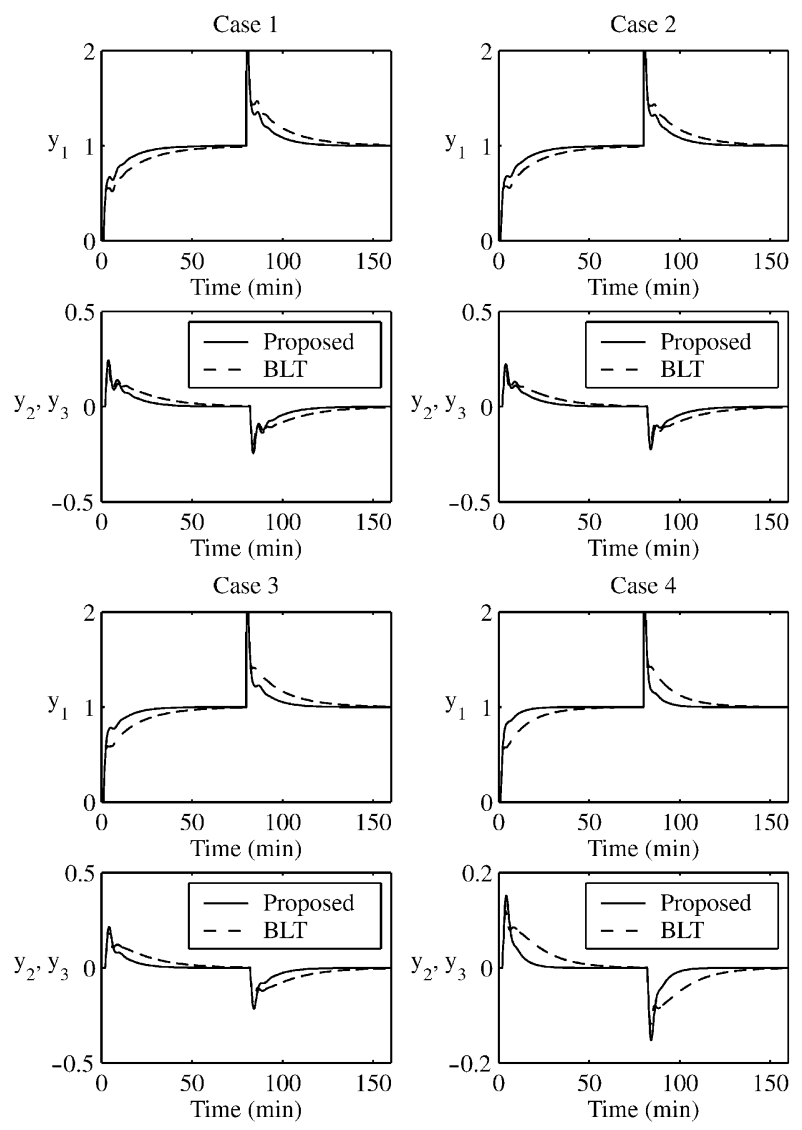

Fig. $5.3 \times 3$ system, cases $1-4$. where $X_{\mathrm{D}}$ and $X_{\mathrm{B}}$ are the overhead and bottoms mole fractions of methanol, respectively; $R$ is the reflux flow rate and $S$ is the steam flow rate to the reboiler; $F$ is the feed flow rate, a disturbance variable.

The stability boundary shown in Fig. 3 for the $l$-th PI controller was obtained by taking the intersection of the column diagonal dominance region from Eq. (14) and the individual stability region from Eq. (15). Six decentralized PI controller settings reported in the literature are given in Table 1 and shown in Fig. 3.

All of these controller settings are located inside the stability region except the ones for Method 2. As discussed in Section 1, the stability condition is a sufficient but not necessary condition. Lee et al. [8] have developed a phase stability condition which makes their stability conditions less conservative. Therefore, it is not surprising that their settings are just outside the stability region obtained here. Although the stability conditions proposed by Lee et al. [8] may provide less conservative results, their method is very complicated to apply. Thus, it can be concluded that the simple stability condition presented in the present paper provides a suitable stability boundary for decentralized PI controller design.
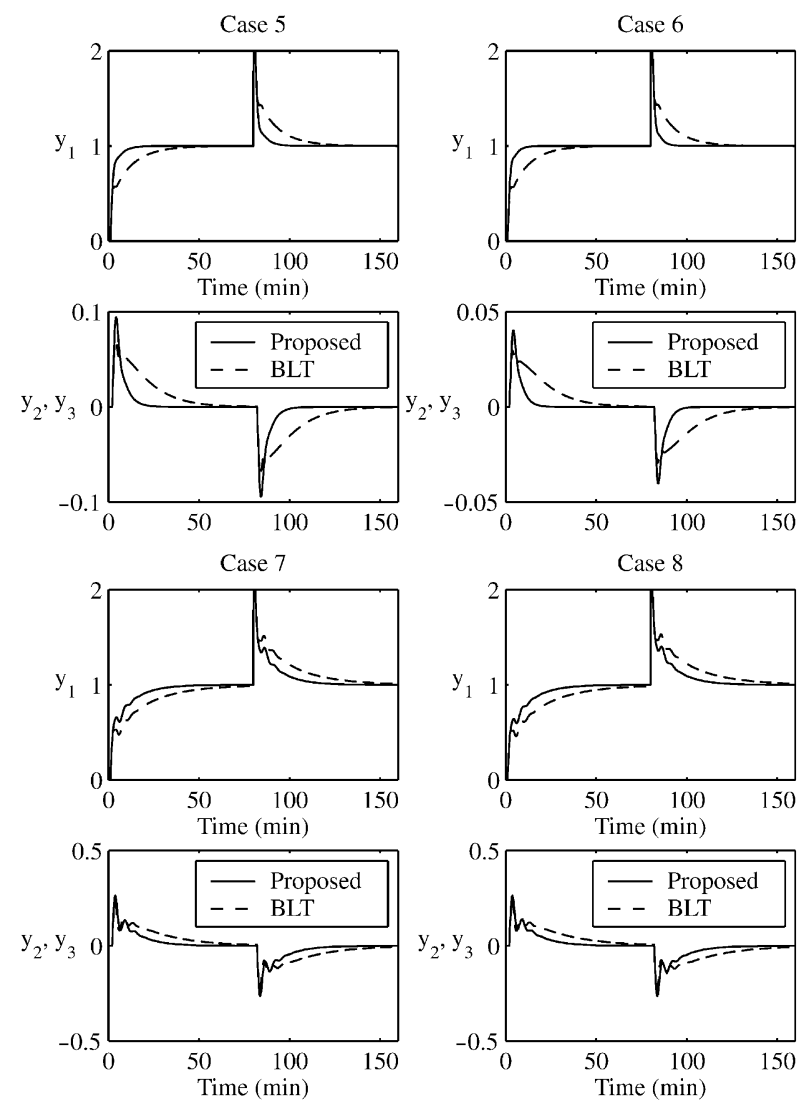

Fig. $6.3 \times 3$ system, cases $5-8$. 


\section{Tuning method for decentralized PI controllers}

The stability region calculation of the previous section provides a stability boundary for the parameters of each PI controller. Thus closed-loop stability is guaranteed for any controller settings that lie inside the stability region. However, the performance of the closed-loop system may vary significantly for different PI parameter
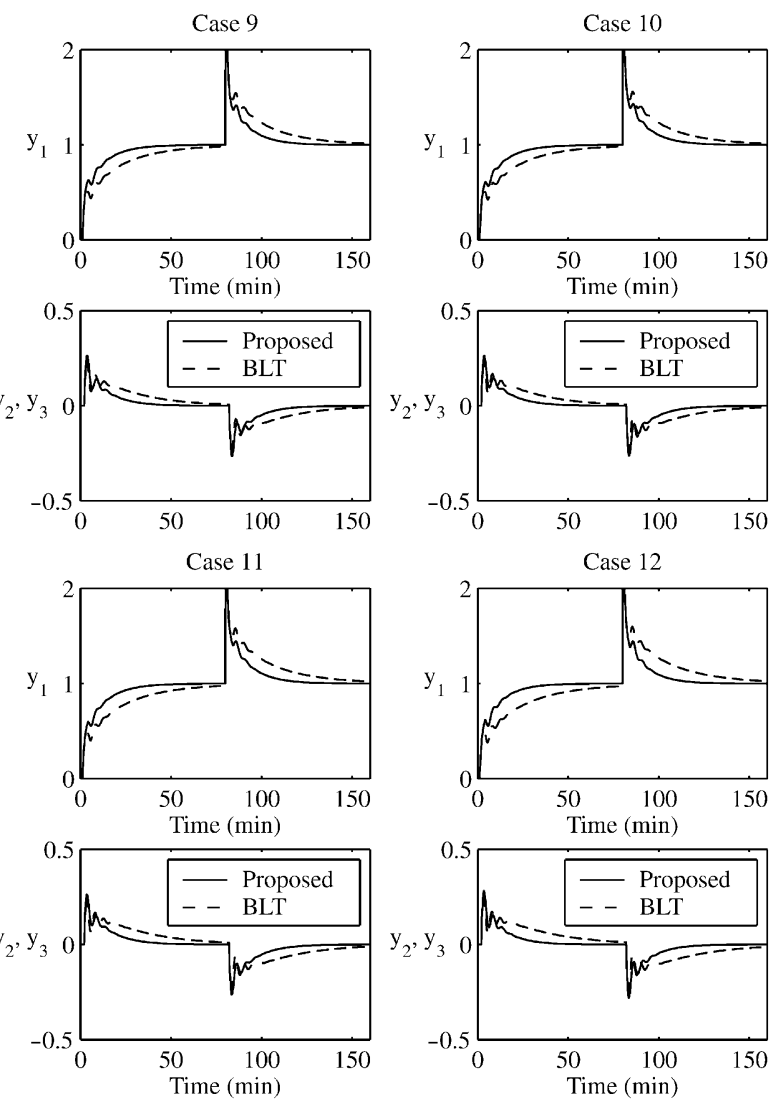

Fig. 7. $3 \times 3$ system, cases 9-12.
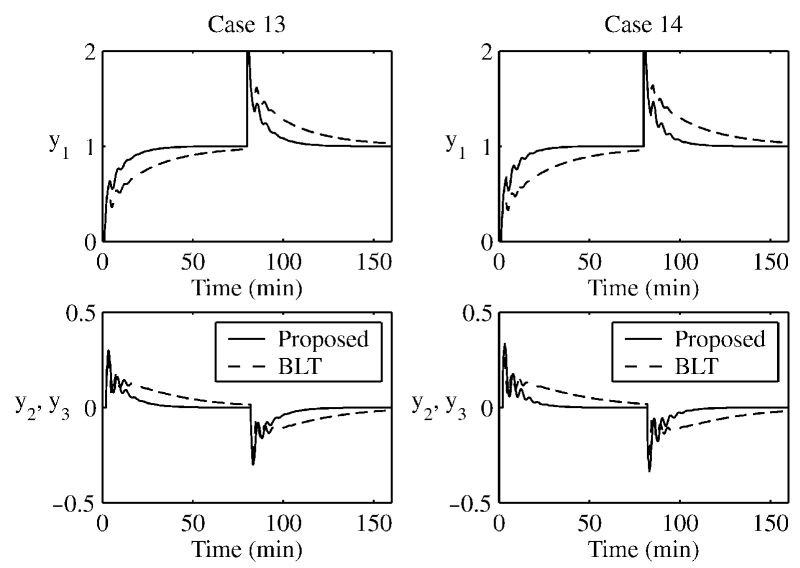

Fig. $8.3 \times 3$ system, cases 13-14 settings. Next, we propose a tuning procedure to choose appropriate controller settings for each loop.

The first step in the proposed tuning method is to calculate the stability region for each PI controller. The ultimate gain $K_{u l}$ of the $l$-th loop [20] is the $K_{c l}$ with the maximum magnitude of $\left|K_{c l}\right|$ on the stability boundary. The corresponding frequency is defined as the ultimate frequency of the $l$-th loop, $\omega_{u l}$. The ultimate gain and ultimate frequency can be obtained from the calculation of stability region using Eqs. (14) and (15). Analytical formulas for calculating the ultimate gain and ultimate frequency were developed in Chen and Seborg [20]. This ultimate gain $\mathbf{K}_{u l}$ and ultimate frequency $\omega_{u l}$ of the $l$-th loop are different from those calculated for the diagonal transfer function $g_{l l}(j \omega)$ in the classical SISO approach because the process interactions are included in the proposed method.

Next, a detuning factor $\mathrm{F}_{l}$ for the $\mathrm{ZN}$ tuning relations is calculated for each control loop. The column diagonal dominance information should be taken into account because the radii of the Gershgorin bands, and thus the stability regions, are directly related to the column diagonal dominance. A column diagonal dominance index for each loop is defined as,

$\phi_{l}(\omega) \triangleq \frac{\left|g_{l l}(j \omega)\right|-\sum_{k=1, k \neq l}^{n}\left|g_{k l}(j \omega)\right|}{\left|g_{l l}(j \omega)\right|}=1-\frac{R_{l} \omega}{r_{l l}(\omega)}$

At each frequency, $\phi_{l}(\omega)$ is a real number that is less than or equal to one. Processes with more severe loop interactions have smaller $\phi_{l}(\omega)$ values. Let $\phi_{u l}$ denote the column diagonal dominance index at the ultimate frequency $\omega_{u l}$ :

$\phi_{u l}=\phi_{l}\left(\omega_{u l}\right)$

In order to develop a tuning criterion for calculating the detuning factor $F_{l}$ from the column diagonal dominance index $\phi_{u l}, 14$ different $3 \times 3$ systems with a large range of $\phi_{u l}$ values have been evaluated. All of the $3 \times 3$ systems have the same structure: the transfer function matrix is symmetric and the diagonal elements are the same. The diagonal elements $g_{i i}(s)$ and the off-diagonal elements $g_{i j}(s)$ are shown in Table 2. Because the transfer function matrix is symmetric, the $\phi_{u l}$ value is the same for all loops of each case. Therefore, for each of

Table 3

Wood-Berry column example

\begin{tabular}{llllllll}
\hline Loop $l$ & $\phi_{u l}$ & \multirow{2}{*}{$F_{l}$} & \multicolumn{2}{c}{ Proposed design } & & \multicolumn{2}{c}{ BLT design } \\
& & & $K_{c l}$ & $\tau_{l l}$ & & $K_{c l}$ & $\tau_{I l}$ \\
\hline$X_{\mathrm{D}}$ & 0.212 & 0.447 & 0.436 & 11.0 & & 0.375 & 8.29 \\
$X_{\mathrm{B}}$ & 0.328 & 0.418 & -0.0945 & 15.5 & & -0.075 & 23.6 \\
\hline
\end{tabular}


the 14 cases, the same values of $F_{l}, K_{c l}$, and $\tau_{I l}$ are obtained for each of the three loops. Furthermore, the Relative Gain Array (RGA) element, $\lambda_{11}$ [28,29], for each case is shown in Table 2. The RGA element is a commonly used index for steady-state loop interaction, but obviously it is not a good index for measuring diagonal dominance. These 14 systems have large differences in column diagonal dominance, but the values of the RGA element, $\lambda_{11}$, are very similar for these cases.
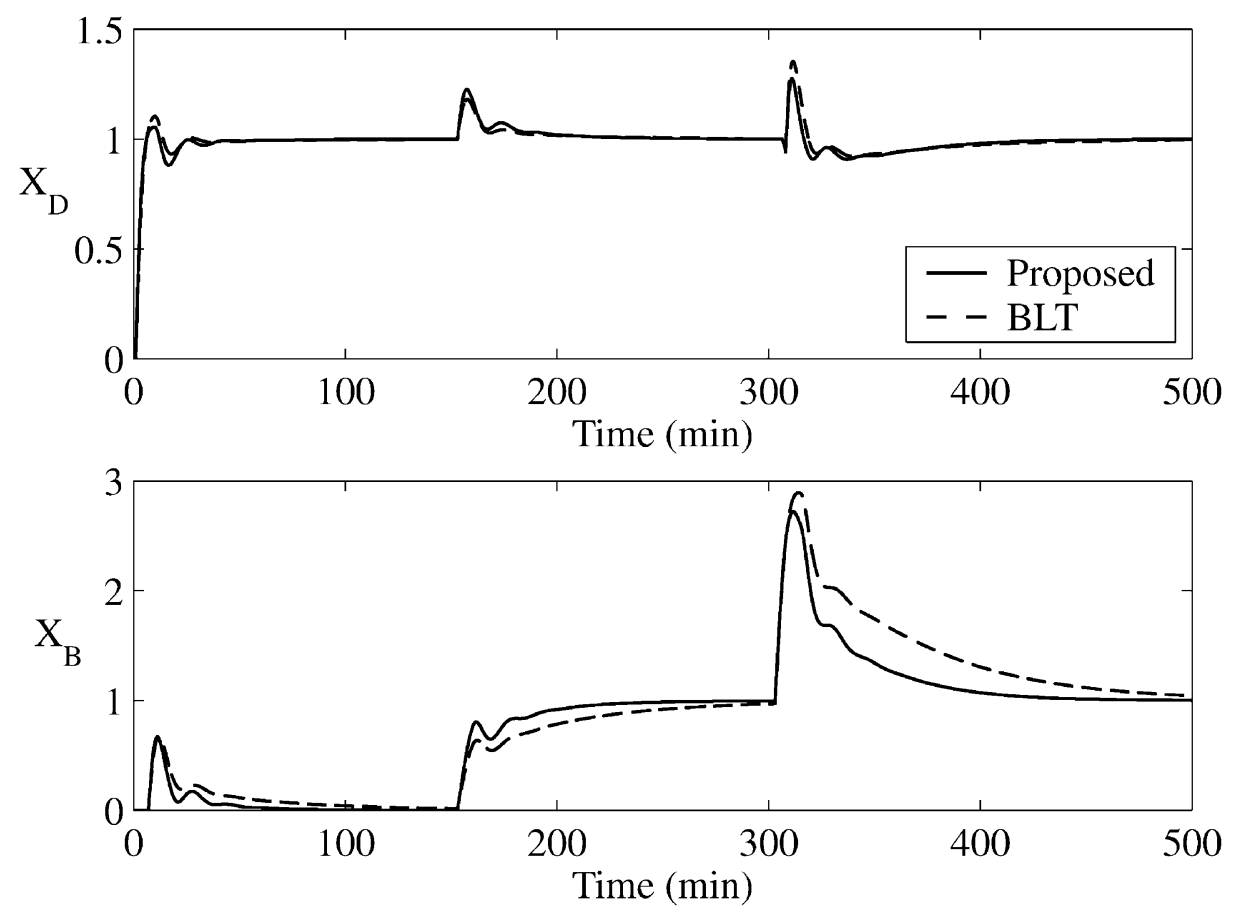

Fig. 9. Closed-loop responses for the Wood-Berry column example.
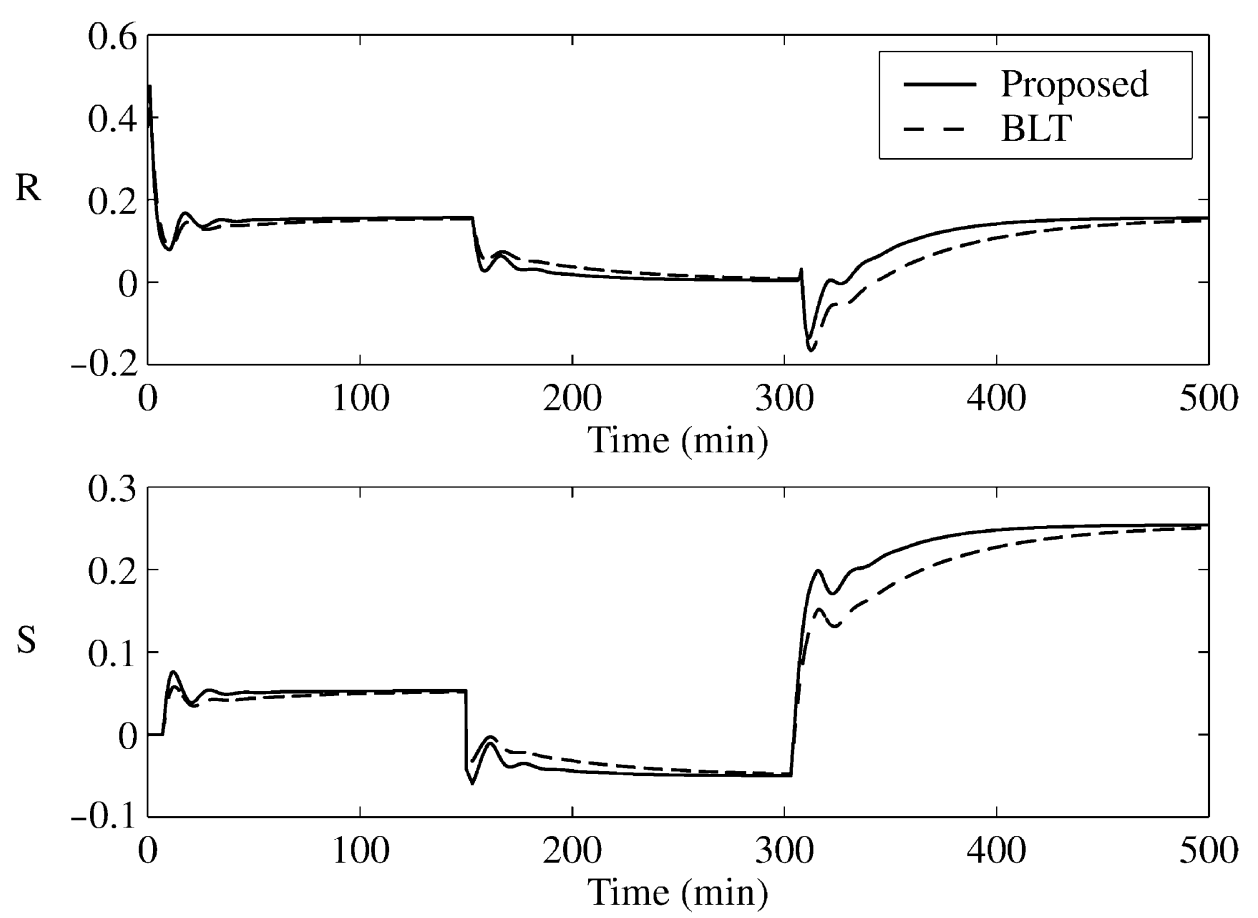

Fig. 10. Controller outputs for the Wood-Berry column example. 
Based on analysis and simulation results for the 14 cases, the following tuning relation is proposed for the $\mathrm{F}_{l}$ factor and the column diagonal dominance index $\phi_{u l}$,

$F_{l}= \begin{cases}0.75 & \text { if } \phi_{u l} \leqslant-1.5 \\ 0.375-0.25 \phi_{u l} & \text { if }-1.5<\phi_{u l} \leqslant-0.5, \\ 0.5 & \text { if }-0.5<\phi_{u l} \leqslant 0, \\ 0.5-0.25 \phi_{u l} & \text { if } 0<\phi_{u l} \leqslant 1\end{cases}$

Because $\phi_{u l} \leqslant 1, F_{l}$ varies between 0.25 and 0.75 .

Finally, the proportional gain of the $l$-th PI controller, $K_{c l}$, is calculated by multiplying the ultimate gain $K_{u l}$ by $F_{l}$,

$K_{c l}=K_{u l} F_{l}$

For this $K_{c l}$, the corresponding value of $K_{I l}$ that lies on the stability boundary is denoted as $K_{I l}^{*}$, as shown in Fig. 4. Then the recommended value of the integral gain is,

Table 4

Vinante-Luyben column example

\begin{tabular}{|c|c|c|c|c|c|c|c|c|}
\hline \multirow[t]{2}{*}{ Loop $l$} & \multirow[t]{2}{*}{$\phi_{u l}$} & \multirow[t]{2}{*}{$F_{l}$} & \multicolumn{2}{|c|}{$\begin{array}{l}\text { Proposed } \\
\text { design }\end{array}$} & \multicolumn{2}{|c|}{ BLT 1} & \multicolumn{2}{|l|}{ BLT 2} \\
\hline & & & $K_{c l}$ & $\tau_{I l}$ & $K_{c l}$ & $\tau_{I l}$ & $K_{c l}$ & $\tau_{I l}$ \\
\hline 1 & -0.666 & 0.542 & 1.21 & 4.64 & 2.08 & 5.96 & -1.07 & 7.1 \\
\hline 2 & 0.440 & 0.390 & 3.74 & 1.10 & 6.38 & 2.22 & 1.97 & 2.58 \\
\hline
\end{tabular}

$$
K_{I l}=K_{I l}^{*} F_{l}
$$

and the corresponding integral time of controller $c_{l}(s)$ is calculated as,

$\tau_{I l}=\frac{K_{c l}}{K_{I l}}$

Because the detuning factor $F_{l}$ is limited to values less than one, the PI controller settings of each loop are located inside the stability regions. Thus closed-loop stability is guaranteed for the resulting PI controllers. In the proposed tuning method, different detuning factors can be obtained for each loop due to the different degrees of column diagonal dominance of each loop, while the BLT method uses the same detuning factor for all loops.

The proposed tuning method has been applied to the $143 \times 3$ systems in Table 2 . All 14 systems are openloop, column diagonally dominant, and thus a decoupler is not required. The column diagonal dominance index $\phi_{u l}$, the detuning factor Fl, and the PI controller settings for the proposed method and the BLT method are also shown in Table 2. The corresponding simulation results are shown in Figs. 5-8 for a $y_{1}$ set-point change followed by a unit step disturbance at the inputs at $t=80 \mathrm{~min}$. Note that the $y_{2}$ and $y_{3}$ responses are symmetrical due to the symmetry of $\mathbf{G}(s)$. In general, the proposed method provides better control because its settling times are shorter.
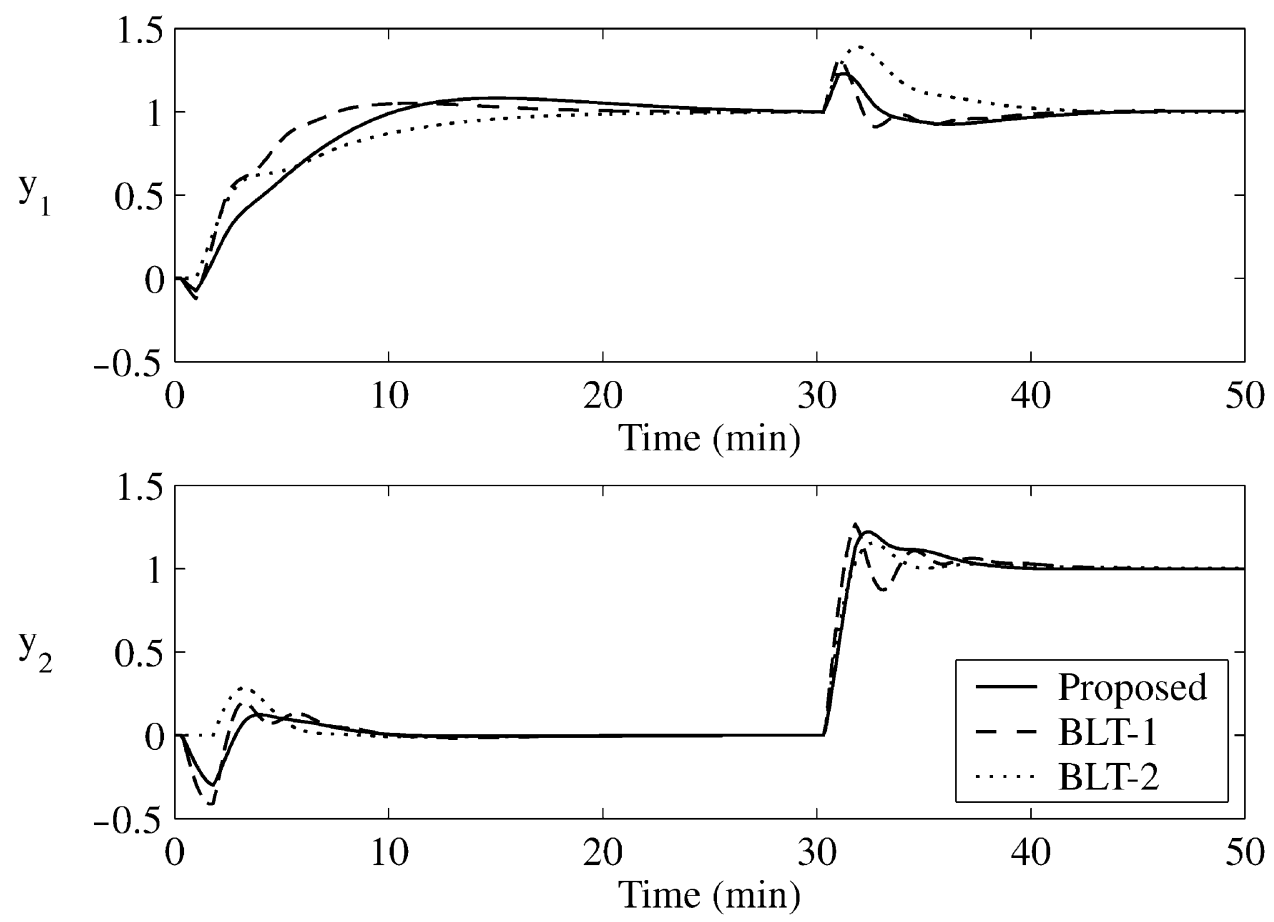

Fig. 11. Set-point change responses for the Vinante-Luyben column example. 

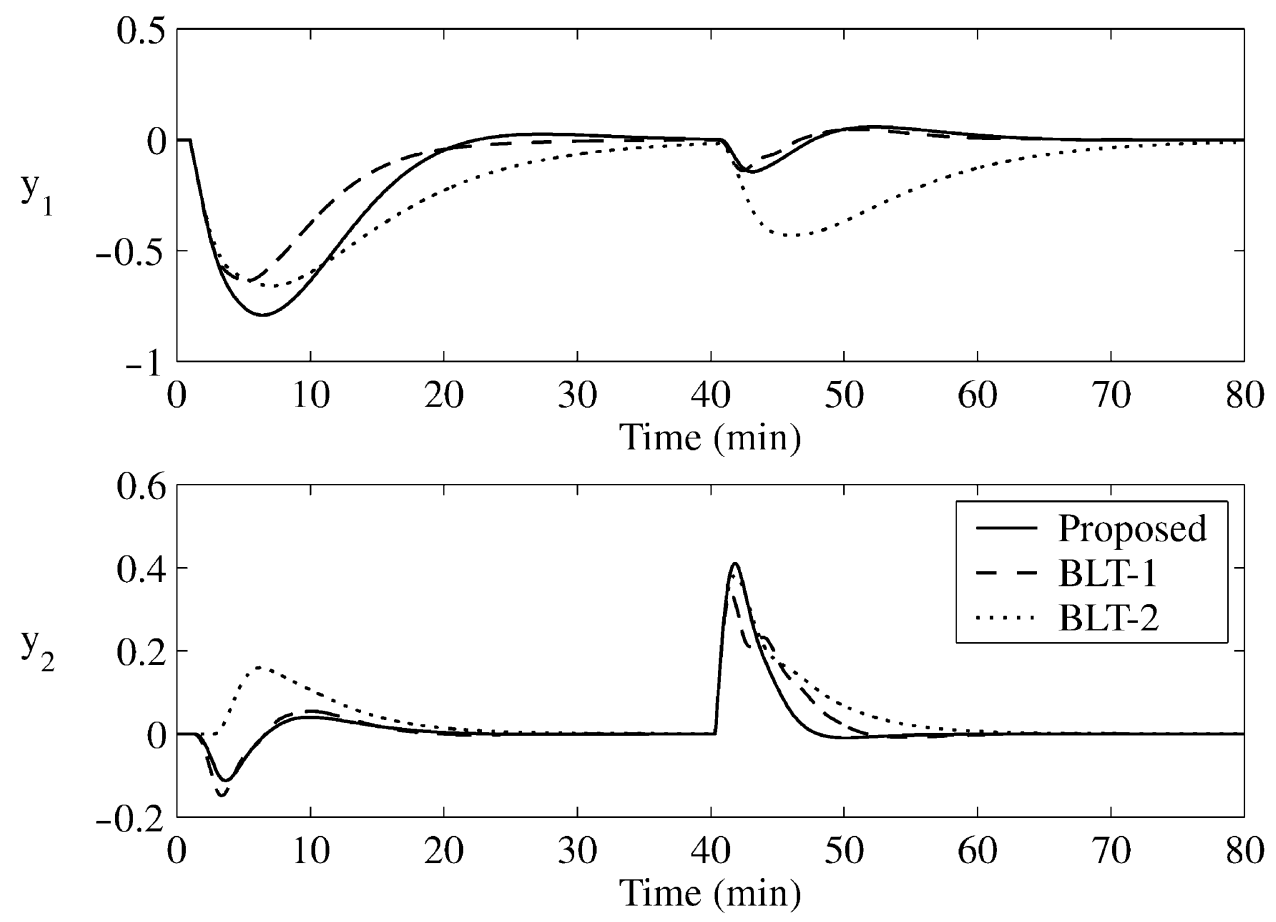

Fig. 12. Disturbance responses for the Vinante-Luyben column example.

\section{Simulation examples}

In this section, two $2 \times 2$ systems and a $4 \times 4$ system considered by Luyben [4] are considered to demonstrate the proposed method.

\subsection{Example 1. Wood-Berry distillation column model}

The Wood-Berry column model in Eq. (16) is openloop, column diagonally dominant. Therefore, a static decoupler was not required. First, the stability regions shown in Fig. 3 were calculated. The column diagonal dominance index $\phi_{u l}$ and the corresponding detuning factor $F_{l}$ for each loop are shown in Table 3. By applying the proposed method, two PI controllers were designed and are compared to the BLT settings in Table 3.

The closed-loop responses for unit step changes in the set-points for $X_{\mathrm{D}}$ (at $\left.t=0\right)$ and $X_{\mathrm{B}}($ at $t=150 \mathrm{~min})$ and for a unit step feed ow disturbance at $t=300 \mathrm{~min}$ are shown in Fig. 9. Fig. 10 shows that the controller outputs are similar for both methods. The $X_{\mathrm{D}}$ responses of the proposed design method and the BLT method are similar, but the $X_{\mathrm{B}}$ responses of the proposed method are better.

\subsection{Example 2. Vinante and Luyben (VL) column}

The transfer function matrix for the VL column system [4] is given by,

$$
\mathbf{G}(s)=\left[\begin{array}{cc}
\frac{-2.2 e^{-s}}{7 s+1} & \frac{1.3 e^{-0.3 s}}{7 s+1} \\
\frac{-2.8 e^{-1.8 s}}{9.5 s+1} & \frac{4.3 \mathrm{e}^{-0.3 s}}{9.2 s+1}
\end{array}\right]
$$

From the transfer function matrix, it is easy to tell that the VL column system does not exhibit open-loop column diagonal dominance. Therefore, a static decoupler, $\mathbf{D}(0)=\mathbf{G}^{-1}(0)$, was applied and the stability region for each loop $\left(K_{c l}, K_{I l}\right)$ was calculated for the augmented system, $\mathbf{G}(s) \mathbf{D}(0)$. Using the proposed tuning method, the $\phi_{u l}, F_{l}$ and the PI controller settings for the augmented system were calculated and are compared to the BLT settings in Table 4. The BLT method was applied to design PI controllers for both the augmented system and the original system. Thus, two sets of PI settings

Table 5

Alatiqi column (A1) example

\begin{tabular}{|c|c|c|c|c|c|c|}
\hline \multirow[t]{2}{*}{ Loop $l$} & \multirow[t]{2}{*}{$\phi_{u l}$} & \multirow[t]{2}{*}{$F_{l}$} & \multicolumn{2}{|c|}{ Proposed design } & \multicolumn{2}{|c|}{ BLT design } \\
\hline & & & $K_{c l}$ & $\tau_{I l}$ & $K_{c l}$ & $\tau_{I l}$ \\
\hline 1 & -8.41 & 0.750 & 0.176 & 62.9 & 0.881 & 85.4 \\
\hline 2 & -2.87 & 0.750 & 0.220 & 31.0 & 4.14 & 6.74 \\
\hline 3 & 0.777 & 0.306 & 3.15 & 8.03 & 2.86 & 9.59 \\
\hline 4 & -0.665 & 0.541 & 0.447 & 47.5 & 0.524 & 94.9 \\
\hline
\end{tabular}



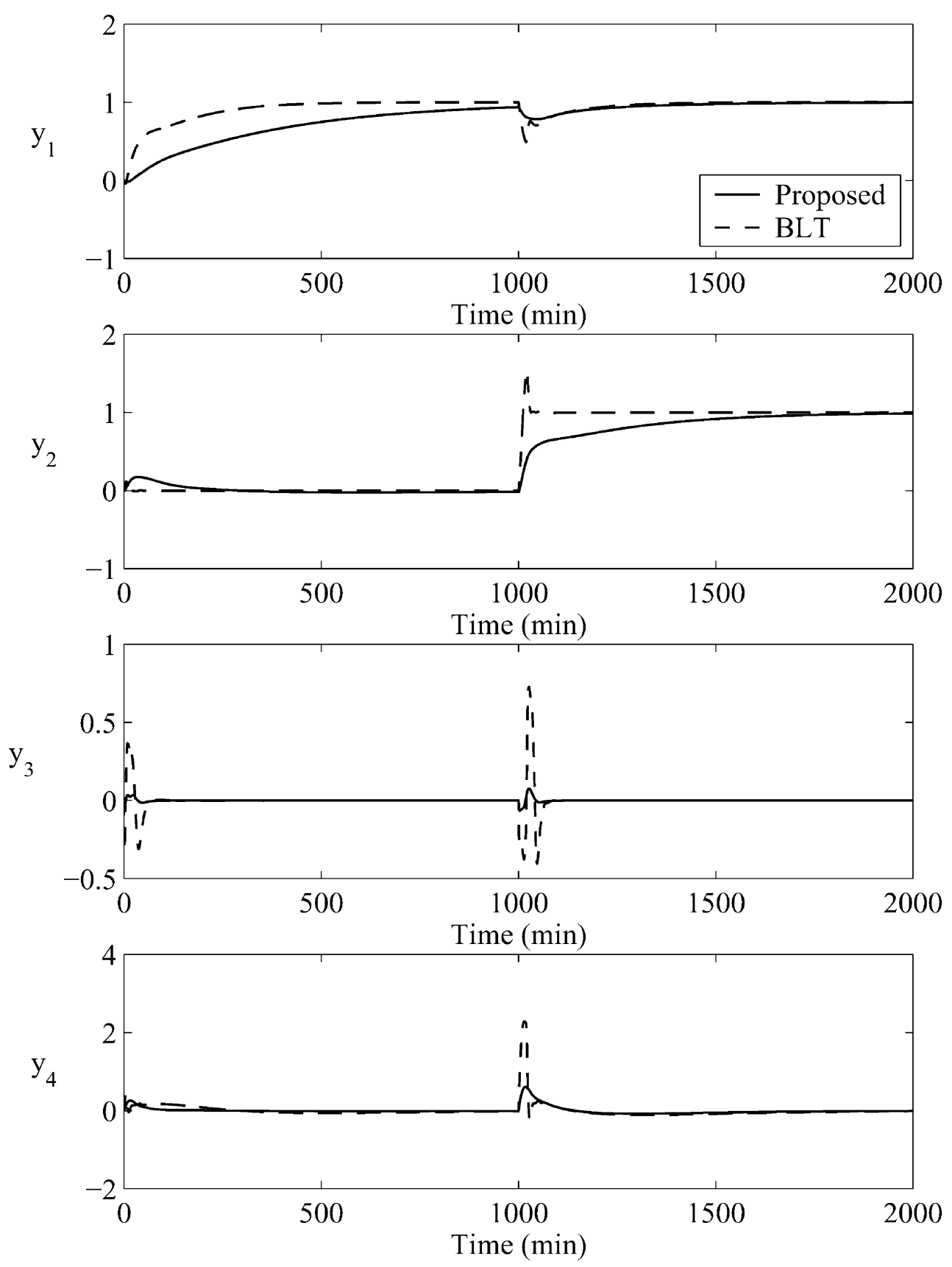

Fig. 13. Responses for set-point changes on $y_{1}$ and $y_{2}$ : Alatiqi column (A1) example.

were obtained: BLT-1 settings are for the augmented system (i.e., the static decoupler is used) and BLT-2 settings are for the original system with no decoupler.

Unit step changes were introduced in the set-points for $y_{1}($ at $t=0)$ and $y_{2}$ (at $t=30 \mathrm{~min}$ ). The closed-loop responses are shown in Fig. 11. The simulation results indicate that the set-point responses of the proposed PI controllers are less oscillatory than those of the BLT-1 PI controllers. The responses for unit step disturbances are shown in Fig. 12. A step change occurred in $u_{1}$ at $t=0$ and in $u_{2}$ at $t=40 \mathrm{~min}$. For these input dis- turbances, the proposed PI controllers provide a slower $y_{1}$ response but a faster $y_{2}$ response in comparison with the BLT-1 PI controllers. When no static decoupler is included, the resulting BLT-2 controllers give slower closed-loop responses. In particular, the disturbance responses of the BLT-2 controllers are very sluggish.

\subsection{Example 3. Alatiqi column (A1)}

The transfer function matrix $\mathbf{G}(s)$ for the A1 column system [4] is given by, 
$\frac{-2.94(7.9 s+1) e^{-0.05 s}}{(23.7 s+1)^{2}}$

$\frac{0.017 e^{-0.2 s}}{(31.6 s+1)(7 s+1)}$

$\frac{3.46 e^{-1.01 s}}{32 s+1}$

$$
\frac{3.511 e^{-13 s}}{(12 s+1)^{2}}
$$$$
\overline{(12 s+1)^{2}}
$$

$\frac{4.32(25 s+1) e^{-0.01 s}}{(50 s+1)(5 s+1)}$ $\frac{-0.51 e^{-7.5 s}}{(32 s+1)^{2}}$

$$
\underline{4.41 e^{-1.01 s}}
$$$$
\overline{16.2 s+1}
$$

$\frac{-1.25 e^{-2.8 s}}{(43.6 s+1)(9 s+1)}$ $\frac{-0.64 e^{-20 s}}{(29 s+1)}$ $\frac{1.68 e^{-2 s}}{(28 s+1)^{2}}$$$
\frac{-5.38 e^{-0.5 s}}{17 s+1}
$$
$\left.\frac{4.78 e^{-1.15 s}}{(48 s+1)(5 s+1)}\right]$
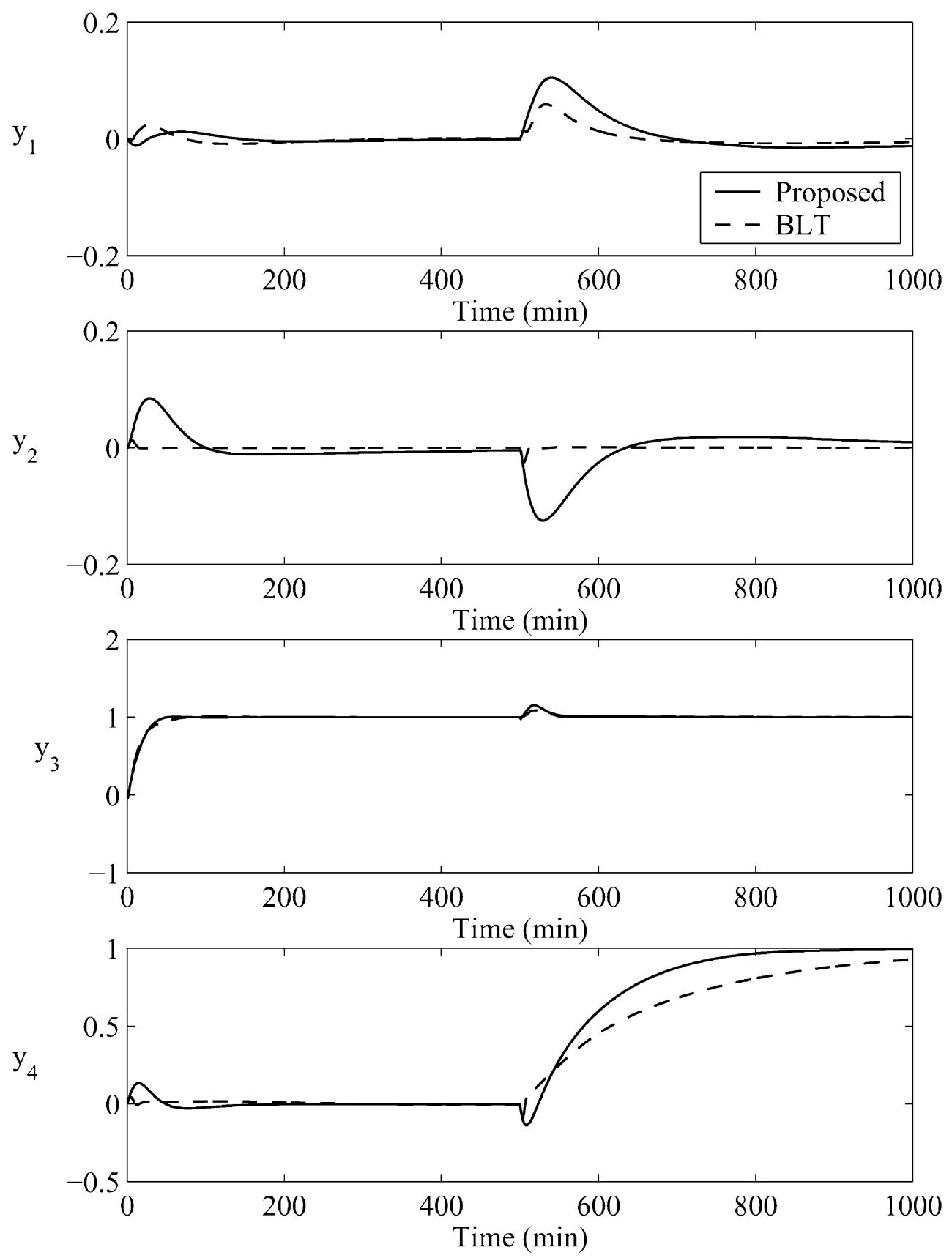

Fig. 14. Responses for set-point changes on $y_{3}$ and $y_{4}$ : Alatiqi column (A1) example. 
Because this system does not exhibit open-loop column diagonal dominance, a static decoupler, $\mathbf{D}(0)=\mathbf{G}^{-1}(0)$, was applied. The stability region for each loop $\left(K_{c l}, K_{I l}\right)$ was obtained for the augmented system, $\mathbf{G}(s) \mathbf{D}(0)$. Table 5 gives the $\phi_{u l}, F_{l}$ and the PI controller settings for the augmented systems obtained using the proposed method. The BLT method was also used to obtain the PI controller settings for the augmented system.

Fig. 13 shows the responses for unit step changes in the set-points of $y_{1}$ (at $\left.t=0\right)$ and $y_{2}$ (at $t=1000 \mathrm{~min}$ ). The responses for unit step changes in the set-points of $y_{3}($ at $t=0)$ and $y_{4}$ (at $t=500 \mathrm{~min}$ ) are given in Fig. 14. The simulation was implemented with saturation limits imposed on each of the input variables: $-1 \leqslant u_{i} \leqslant 1$ for all $i=1, \ldots, 4$. The simulation results indicate that the proposed design method provides faster responses with smaller overshoot for loop 3 and loop 4 than the BLT method, but the responses of loops 1 and 2 for the proposed method are much more sluggish. Table 5 shows that the A1 column has larger negative $\phi_{u l}$ values for loops 1 and 2. Thus, it can be concluded that the proposed method may be conservative for highly interacting systems, i.e., systems with large negative $\phi_{u l}$ values.

\section{Conclusions}

The stability of decentralized PI control systems has been analyzed based on Nyquist stability analysis. In particular, a stability region has been derived for each PI controller from system frequency response information. A tuning method has been proposed based on the obtained stability region and a new column diagonal dominance index for each loop. The resulting decentralized control system can guarantee closed-loop stability. Simulation results illustrate that this design method provides good performance for a wide range of examples.

\section{Acknowledgements}

Financial support from the UCSB Process Control Consortium is gratefully acknowledged. A preliminary version of this paper was presented at the IEEE Conference on Control Applications, Mexico City (September, 2001).

\section{References}

[1] K.J. Åström, T.H. Hägglund, PID Controllers: Theory, Design, and Tuning, second ed, Instrument Society of America, Research Triangle Park, NC, 1995.

[2] K.J. Åström, T. Hägglund, The future of PID control, Control Engineering Practice 9 (2001) 1163-1175.
[3] G.K. McMillan, Tuning and Control Loop Performance, third ed, Instrument Society of America, Re-search Triangle Park, NC, 1994.

[4] W.L. Luyben, Simple method for tuning SISO controllers in multivariable systems, Ind. Eng. Chem. Des. Dev 25 (1986) 654-660.

[5] T.J. Monica, C.-C. Yu, W.L. Luyben, Improved multiloop single-input/single-output (SISO) controllers for multivariable processes, Ind. Eng. Chem. Res. 27 (1988) 969-973.

[6] W.L. Luyben, M.L. Luyben, Essentials of Process Control, McGraw-Hill, New York, 1997.

[7] W.K. Ho, T.H. Lee, O.P. Gan, Tuning of multiloop proportional-integral-derivative controllers based on gain and phase margin specifications, Ind. Eng. Chem. Res. 36 (1997) 2231-2238.

[8] J. Lee, W. Cho, T.F. Edgar, Multiloop PI controller tuning for interacting multivariable processes, Computers Chem. Eng. 22 (1998) 1711-1723.

[9] C.C. Yu, Autotuning of PID Controllers: Relay Feedback Approach, Springer-Verlag, New York, 1999.

[10] Y. Halevi, Z.J. Palmor, T. Efrati, Automatic tuning of decentralized PID controllers for MIMO processes, J. Process Control 7 (1997) 119-128.

[11] A.P. Loh, C.C. Hang, C.K. Quek, V.U. Vasnani, Autotuning of multiloop proportional-integral controllers using relay feedback, Ind. Eng. Chem. Res. 32 (1993) 1102-1107.

[12] A.P. Loh, V.U. Vasnani, Describing function matrix for multivariable systems and its use in multiloop PI design, J. Process Control 4 (1994) 115-120.

[13] S.-H. Shen, C.-C. Yu, Use of relay-feedback test for automatic tuning of multivariable systems, AIChE J. 40 (1994) 627-646.

[14] Z.J. Palmor, Y. Halevi, N. Krasney, Automatic tuning of decentralized PID controllers for TITO processes, Automatica 31 (1995) 1001-1010

[15] J.G. Ziegler, N.B. Nichols, Optimum settings for automatic controllers, Trans. ASME 64 (1942) 759-768.

[16] K.K. Tan, Q.-G. Wang, C. Hang, Advances in PID Control, Springer-Verlag, New York, 1999.

[17] C.C. Hang, K.J. Åström, W.K. Ho, Refinements of the ZieglerNichols tuning formula, IEE Proc.-D 138 (1991) 111-117.

[18] D.J. Hawkins, Pseudodiagonalisation and the inverse Nyquist array method, Proc. IEE 119 (1972) 337-342.

[19] M.P. Ford, K.C. Daly, Dominance improvement by pseudodecoupling, Proc. IEE 126 (1979) 1316-1320.

[20] D. Chen, D. E. Seborg, Multiloop PI/PID controller design based on Gershgorin bands, IEE Proc.-Control Theory Appl. 149 (2002) 68-73.

[21] D. Chen, D.E. Seborg, Robust Nyquist array analysis based on uncertainty descriptions from system identification, Automatica 38 (2002) 467-475.

[22] D. Chen, Design and Analysis of Decentralized PID Control Systems, PhD Thesis, University of California Santa Barbara, 2002.

[23] P. Grosdidier, M. Morari, Interaction measures for systems under decentralized control, Automatica 22 (1986) 309-319.

[24] H.H. Rosenbrock, Computer-Aided Control System Design, Academic Press, New York, 1974.

[25] J.M. Maciejowski, Multivariable Feedback Design, AddisonWesley, Reading, MA, 1989.

[26] K.J. Åström, H. Panagopoulos, T. Hägglund, Design of PI controllers based on non-convex optimization, Automatica 34 (1998) 585-601.

[27] R.K. Wood, M.W. Berry, Terminal composition control of a binary distillation column, Chem. Eng. Sci. 28 (1973) 1707-1717.

[28] E.H. Bristol, On a new measure of interactions for multivariable process control, IEEE Trans. on Auto. Control AC- 11 (1966) $133-134$.

[29] T.J. McAvoy, Interaction Analysis, Instrument Society of America, Research Triangle Park, NC, 1983.

[30] J. Lee, J.Y. Choi, Design of multiloop PI controller, J. KIChE 31 (1993) 272-278. 